This is the final peer-reviewed accepted manuscript of

Bodner, Martin; luvaro, Alessandra; Strobl, Christina; Nagl, Simone; Huber, Gabriela; PELOTTI, SUSI;

PETTENER, DAVIDE; LUISELLI, DONATA; Parson, Walther: Helena, the hidden beauty: Resolving the most common West Eurasian mtDNA control region haplotype by massively parallel sequencing an Italian population sample. FORENSIC SCIENCE INTERNATIONAL: GENETICS 15, p. 21-26. ISSN 1872-4973

DOI: 10.1016/j.fsigen.2014.09.012

The final published version is available online at:

http://dx.doi.org/10.1016/i.fsigen.2014.09.012

Rights / License: The terms and conditions for the reuse of this version of the manuscript are specified in the publishing policy. For all terms of use and more information see the publisher's website. 


\title{
Helena, the hidden beauty: Resolving the most common West Eurasian mtDNA control region haplotype by massively parallel sequencing an Italian population sample
}

\author{
Martin Bodner ${ }^{\mathrm{a}}$, Alessandra Iuvaro ${ }^{\mathrm{a}, \mathrm{b}}$, Christina Strobl ${ }^{\mathrm{a}}$, Simone Nagl ${ }^{\mathrm{a}}$, Gabriela Huber ${ }^{\mathrm{a}}$, \\ Susi Pelotti ${ }^{\mathrm{b}}$, Davide Pettener ${ }^{\mathrm{c}}$, Donata Luiselli ${ }^{\mathrm{c}, *}$, Walther Parson ${ }^{\mathrm{a}, \mathrm{d}, * *}$ \\ a Institute of Legal Medicine, Innsbruck Medical University, Innsbruck, Austria \\ ${ }^{\mathrm{b}}$ Department of Medical and Surgical Sciences, Institute of Legal Medicine, University of Bologna, Bologna, Italy \\ ${ }^{\mathrm{c}}$ Department of Biological, Geological and Environmental Science, Laboratory of Molecular Anthropology, University of Bologna, Bologna, Italy \\ d Penn State Eberly College of Science, University Park, PA, USA
}

\begin{abstract}
A B S T R A C T
The analysis of mitochondrial (mt)DNA is a powerful tool in forensic genetics when nuclear markers fail to give results or maternal relatedness is investigated. The mtDNA control region (CR) contains highly condensed variation and is therefore routinely typed. Some samples exhibit an identical haplotype in this restricted range. Thus, they convey only weak evidence in forensic queries and limited phylogenetic information. However, a CR match does not imply that also the mtDNA coding regions are identical or samples belong to the same phylogenetic lineage. This is especially the case for the most frequent West Eurasian CR haplotype 263G 315.1C 16519C, which is observed in various clades within haplogroup H and occurs at a frequency of 3-4\% in many European populations.

In this study, we investigated the power of massively parallel complete mtGenome sequencing in 29 Italian samples displaying the most common West Eurasian CR haplotype - and found an unexpected high diversity. Twenty-eight different haplotypes falling into 19 described sub-clades of haplogroup $\mathrm{H}$ were revealed in the samples with identical CR sequences. This study demonstrates the benefit of complete mtGenome sequencing for forensic applications to enforce maximum discrimination, more comprehensive heteroplasmy detection, as well as highest phylogenetic resolution.
\end{abstract}

\author{
Keywords: \\ Massively parallel sequencing \\ mtDNA \\ Forensics \\ Most common haplotype \\ Power of discrimination \\ mtDNA haplogroup $\mathrm{H}$
}

\section{Introduction}

Forensic DNA analyses are routinely performed by determining the "genetic fingerprint", i.e. the alleles of polymorphic nuclear microsatellite markers that display high diversity, stability, and Mendelian inheritance, and thus allow identification,

\footnotetext{
Abbreviations: codR, coding region of the mtDNA; CR, control region of the mtDNA EDNAP, European DNA Profiling Group; EMPOP, EDNAP mtDNA population database; MPS, massively parallel sequencing; MRCA, most recent common ancestor; mtDNA, mitochondrial DNA; np(s), nucleotide position(s).

* Corresponding author. Tel.: +39 0512094194; fax: +39 0512094191.

** Corresponding author at: Institute of Legal Medicine, Innsbruck Medical University, Innsbruck, Austria. Tel.: +43 512900370651; fax: +43 512900373640 E-mail addresses: donata.luiselli@unibo.it (D. Luiselli) walther.parson@i-med.ac.at (W. Parson).
}

individualization and pedigree reconstruction [1]. These markers however do not regularly yield results from compromised samples containing degraded or low quantities of nuclear DNA. The haploid, maternally inherited mitochondrial ( $\mathrm{mt}$ )DNA has become a vital niche in analyzing those samples due to its abundance and stability as multi-copy circular molecule protected in organelles. As a lineage marker, it can be used to exclude identity or corroborate (even distant) maternal relatedness [2-6].

The outcome of (forensic) mtDNA investigations, besides precise base calling and the availability of high-quality databases [7-10], mainly depends on the amount of information generated from the individual sample $[11,12]$. Because of financial, technical and legal restrictions, the current standard is to sequence (hypervariable parts of) the $\sim 1.1 \mathrm{kbp}$ non-coding control region (CR) of the $\sim 16.5 \mathrm{kbp}$ mitochondrial genome (mtGenome), that contains densely concentrated variation due to a higher mutation 
rate compared to the remaining segment, the coding region (codR) $[7,13,14]$. CR data enable coarse discrimination of numerous maternal lineages [15] and may yield non-identity ("exclusion") of donors and their maternal relatives, respectively, in many forensic cases $[16,17]$. However, identical CR haplotypes are found on different haplogroup backgrounds across the mtDNA phylogeny, as several lineages are poorly defined in the CR [18]. Consequently, a shared CR haplotype ("non-exclusion") does not necessarily imply that two mtDNAs are identical in their codR, or even belong to the same lineage.

For these reasons, the current partial analyses of the mtDNA molecule greatly restrict and possibly bias (forensic) interpretation, and are particularly problematic for populations with extremely few CR lineages (e.g., Ref. [19]). For example, when working with samples of West Eurasian (sometimes referred to as "Caucasian") maternal background, it is very likely to encounter diverse clades of haplogroup $\mathrm{H}$ that encompasses $\sim 40 \%$ in many European populations [20-22]. The currently available CR data convey a rather uniform picture of distribution, while pivotal studies at higher levels of phylogenetic resolution have demonstrated a cluster of $>100$ distinct radiating lineages within this haplogroup, with significant differences in dispersal and frequency (e.g., Refs. [20,21,23-27]). These investigations have so far mostly focused on only few or non-random samples, involved limited codR sequencing, contained no (detailed) information on the donors' geographic origin or were derived from small regions [10]. Only about one fifth of the $\mathrm{H}$ lineages can be distinguished within the $C R$, but markers are often homoplasic $[14,15,18,23]$ (Fig. 1).

Correspondingly, many West Eurasian individuals exhibit identical CR haplotypes clustering within haplogroup RO (the CR-MRCA of haplogroup $\mathrm{H}$ ) only for the lack of further sequence data. This is particularly the case for the most common West Eurasian CR haplotype 16519C 263G 315.1C (relative to the revised Cambridge Reference Sequence, rCRS [28]), observed in numerous sub-clades of haplogroup $\mathrm{H}$ [15] with a frequency of 3-4\% in any western Eurasian population [22]. It can be anticipated that codR analysis would reveal a high number of different lineages ( $c f .[12,29])$.

Different strategies have been applied to access codR information in forensic casework $[14,30]$ but circumvent laborious complete mtGenome Sanger-type sequencing [6,31,32]. These have included sequencing only short segments comprising variation considered relevant $[33,34]$ or, more commonly, targeting distinct markers of a few principal clades in a (usually minisequencing) multiplex assay in order to determine the main haplogroups on macro-region (e.g., Refs. $[35,36])$ or even global

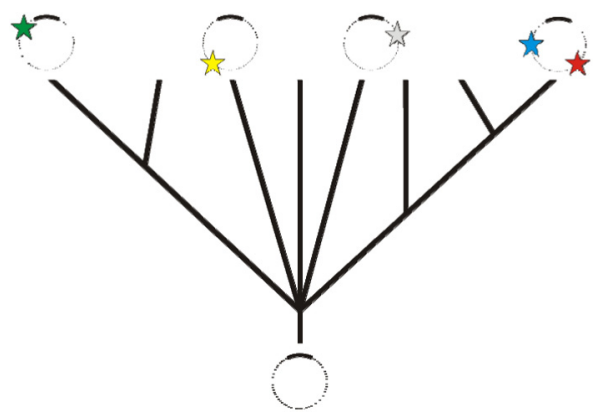

Fig. 1. Schematic "phylotree" of haplogroup H from entire mtGenome sequencing. The root haplogroup $\mathrm{H}$ haplotype (at the bottom) is the origin of five (for the $\sim 100$ ) radiating lineages that give rise to further sub-branches. Four explicative derived $\mathrm{H}$ haplotypes are shown at tips of the tree. If only the mtDNA CR (continuous segments of the circles) were sequenced, all haplotypes in this figure would be identical and would fall into a single $\mathrm{H}^{*}$ lineage. Their individual or lineage diagnostic codR variation, indicated by asterisks in the dotted segments of the circular genomes, would not be detected. level [37-39], or to dissect identical samples or a specific lineage (for haplogroup H, e.g., Refs. [24,40,41] (compared in Ref. [42]), $[43,44])$. The number of markers that can be included in such an assay is limited; therefore, a selection toward those considered more frequent is usually made. In any approach, countless SNPs are left undetermined and consequently a somewhat rough resolution is yielded - only complete mtGenome sequencing would reveal untargeted private or phylogenetic variation in total.

This loss in discrimination power cannot be compensated even when highly fluctuating "individualizing" markers are included in some assays ( $c f$. [42]). Emerging benchtop high-throughput massively parallel (MPS; or next generation) sequencing solutions, that are very promising in terms of sample throughput, speed, amount of data generated and costs per sample, now make obtaining complete mtDNA information relatively easy. In this pilot study, we investigated the power of entire mtGenome MPS on an identical most common West Eurasian mtDNA CR haplotype sample set, carefully considering methodological challenges $[45,46]$ and taking advantage of insights from systematic data reviews during recent complete $\mathrm{mtGenome}$ etalon (i.e. carefully selected high quality reference, $c f$. [47]) dataset generation by both Sanger-type sequencing [6,48] and MPS [49].

\section{Materials and methods}

\subsection{DNA samples}

An earlier population study [44] included 884 randomly selected individuals representing eight macro-areas of Italy that donated blood samples after informed consent. DNA was extracted using a modified salting out method [50], sequenced for mtDNA CR and typed for 22 codR SNPs to determine the main West Eurasian haplogroups. The samples found to belong to haplogroup R0 were further subjected to a SNP multiplex analysis designed to resolve 17 distinct haplogroup $\mathrm{H}$ lineages [44]. In this study, we used a subset of 29 of these samples harboring the most common West Eurasian mtDNA CR haplotype 16519C 263G 315.1C (relative to the rCRS) [28]. Three samples were re-extracted using the QIAamp DNA Mini kit (QIAgen, Hilden, Germany). The donors originated from provinces throughout Italy, including Sicily and Sardinia (Fig. 2 and Table S1).

\subsection{Complete mtDNA sequence data generation and interpretation}

After simultaneous verification of integrity and quantitation of mtDNA in a modular real-time quantitative assay [51], we amplified two overlapping $\sim 8.5 \mathrm{~kb}$ fragments covering the entire mitochondrial genome [31] and performed MPS on the Ion Torrent Personal Genome Machine (PGM) using Ion PGM Sequencing 200 Kit v. 2 chemistry on an Ion 316 chip. Raw data were inspected twice using independent software, mirroring the gold standard in Sanger-type sequencing [7]. The threshold for heteroplasmic mixtures was defined 10\% (of total coverage). We used Sangertype sequencing to clarify discordant positions. Sequences were aligned to the rCRS [28]. According to a basic forensic principle, we compared the MPS results to those from Sanger-type sequencing [44]. We assigned the samples to mtDNA haplogroups according to Phylotree [15], build 16, aided by the EMMA software package [52] and calculated genetic parameters as described in Ref. [53]. We followed strict quality control protocols according to a preceding validation study of the PGM in forensic mtDNA sequencing [46].

\section{Results}

Entire mtGenome sequencing revealed an extremely high degree of variation in the samples that shared the CR motif $16519 \mathrm{C}$ 


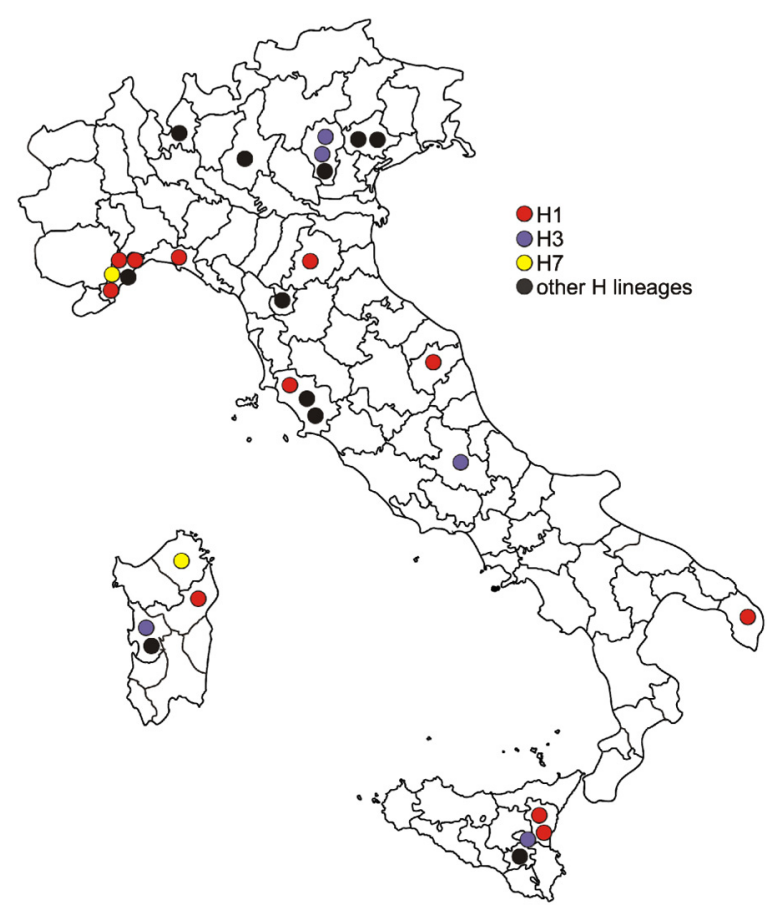

Fig. 2. Origin of the 29 Italian samples included in this study. Circles represent individual samples and are assigned to their province of origin. The color codes distinguish between samples of haplogroups $\mathrm{H} 1, \mathrm{H} 3$, and $\mathrm{H} 7$ and those falling into other $\mathrm{H}$ clades (see text for details). (For interpretation of the references to color in this figure legend, the reader is referred to the web version of the article.)

263G 315.1C. In total, 28 different haplotypes were discerned within the set of 29 samples analyzed in this study. Two (seemingly) unrelated donors from the same province ([44], Table S1), DB525 and DB559, revealed identical mtGenomes. The haplotype diversity using entire $\mathrm{mtGenome}$ information reached $99.8 \%$, corresponding to a random match probability of 0.037 (Table 1$)$. The average MPS coverage along the mtGenomes ranged between 377 and 1154 (median: 687; mean: 697).

The MPS results were fully concordant with the partially available Sanger-type sequencing results [44], with the exception of a low-level point heteroplasmy at np 16311 in sample DB1766 that had not been visible after Sanger-type sequencing, which speaks for an advantageous detection of (heteroplasmic) mixtures with MPS. The 29 complete mtGenomes are reported in Table S1, available from GenBank (accession numbers KM252727KM252755) and will be uploaded onto the EDNAP mtDNA population database (EMPOP V3) [22]. They classified into 19 reported haplogroup $H$ sub-clades ([15], build 16), 17 of which were unique in the dataset. Haplogroups $\mathrm{H} 1$ and $\mathrm{H} 3$ comprised the largest number of mtDNAs (13 and five, respectively) among the

Table 1

Comparison of the diversity parameters in the 29 Italian samples using different sequence ranges.

\begin{tabular}{llll}
\hline & \multicolumn{2}{l}{ mtDNA range } \\
\cline { 2 - 4 } & $\mathrm{CR}$ & $\mathrm{CR}+39$ codR SNPs $^{\mathrm{a}}$ & Complete mtGenome $^{\circ}$ \\
\hline Haplotypes & 1 & 6 & 28 \\
Unique haplotypes $^{\text {CR }}$ & 0 & 2 & 27 \\
Haplogroups $^{\mathrm{b}}$ & 1 & 6 & 20 \\
Unique haplogroups $^{\mathrm{b}}$ & 0 & 2 & 18 \\
RMP $^{\mathrm{c}}$ & 1.000 & 0.296 & 0.037 \\
Haplotype diversity & $0.0 \%$ & $72.9 \%$ & $99.8 \%$ \\
\hline
\end{tabular}

\footnotetext{
a Thereof 17 specific for haplogroup $\mathrm{H}$ clades [44]

b According to Ref. [15], build $16 . \mathrm{H}^{*}$ is considered a haplogroup.

c Random match probability.
}

detected lineages: $\mathrm{H}^{*}(7 / 29=24.1 \%), \mathrm{H}^{*}(4 / 29=13.8 \%)$, and singletons (3.4\%) of H1ax, H1e*, H1e1a, H1j3, H1q*, H1q2, H3ar, H7b*, H7b1, H10a, H18b, H58, H59a, H65, H75, H84 and H86. One sample could not be assigned to a distinct $\mathrm{H}$ lineage (Fig. 3).

In addition to the cytosine insertion after np 315 and the transition at np 16519 (compared to the rCRS [28]) present in all haplotypes but never listed in Phylotree [15], 23 of the samples carried up to four additional, so-called "private" polymorphisms that possibly highlight yet undescribed lineages (Table S1). Two substitutions, T15115G (DB604) and C11588T (DB1368), were previously unobserved. The only recurrent pattern within our dataset was H1-709-8108-15470, pointing at a novel sub-clade in the (seemingly) unrelated samples DB1566 and DB1570 (from the same province [44], and identical aside from a single heteroplasmic position, Table S1). In four instances, unnamed phylogenetic patterns have been reported earlier: H3-2851-11200 in DB604 and sample DQ523661 from Sardinia [54]; H1q-885614258 in DB763, EF657644 from Italy [55] and JX153975 [56], KF161678 and KF162479 [S. Li and M.H. Schierup, unpublished] from Denmark; H1-5581-14905 in DB2633 and JQ703512 [27]; and finally, the transition at np 4245 we found on an haplogroup H7b background in DB1544 was present on an H7 background in EF661013 from Italy [57]. Another phylogeny refinement is highlighted by sample DB1049, where the presence of two diagnostic polymorphisms, viz. transitions at nps 2851 and 14148, indicated haplogroup H1h2 status, while another two currently listed markers, the transitions at nps 7013 and 14420 ([15], build 16), were missing. In sample DB628, the transition at np 5460 would support haplogroup H1e on the H1 background. Based on this single marker, the sample would be misassigned, as three additional SNPs (including a transversion) suggest H1ax status.

\section{Discussion}

\subsection{MtDNA forensics at its highest resolution}

This study strikingly demonstrates the significance of complete mtGenome sequencing in forensic genetic practice: highest mtDNA resolution allowed almost complete discrimination of haplotypes identical in their CR by rendering virtually every one unique in this randomly selected sample. In the previous study that included 39 codR SNPs in addition to the CR [44], a power of discrimination of $72.9 \%$ had been reached using the same sample set, compared to $99.8 \%$ in this study (Table 1). A significant genetic diversity increase when analyzing complete mtGenomes has also been found in larger, randomly chosen population samples with a more ample haplogroup spectrum $[48,49]$.

The two matching complete mtDNA sequences (i.e. "nonexclusion") signpost the current limitation of mtGenome sequencing in forensic applications: these two samples cannot be excluded as deriving from the same individual or the same maternal lineage, respectively. Sound sampling ( $c f$. [58]) and large and reliable sequence databases [10] are necessary prerequisites for a correct assessment of population variation to weigh such evidence. Currently, the forensic community is able to assess the probability of a given haplotype in its (putative) population by using EMPOP [22] (or other databases) in the CR range. Necessary structural and query modifications have recently been elaborated [6], and with the upcoming third version of EMPOP, a complete mtGenome database for forensic application will be accessible ( $c f .[6,10])$. More than 20,000 (nearly-) complete worldwide mtGenome sequences are publicly available (cf. [6,15]), but for now, comprehensive high-quality investigations on randomly selected population samples are still scarce (see above) $[48,49]$. 




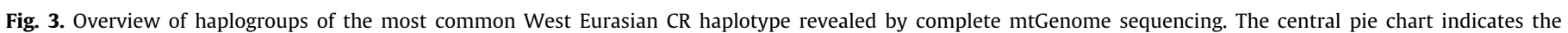

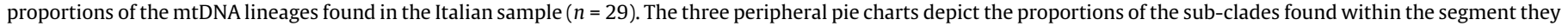
are assigned to. One haplotype does not fall into a described lineage.

\subsection{Helena's many daughters}

Already the small Italian sample analyzed in this study revealed 20 different sub-lineages hiding behind an identical CR sequence. Compared to the previous study [44] and using the same sample set, six of the $\mathrm{H}^{*}$ samples could now be assigned to a specific clade and for ten additional samples (within haplogroups H1, H3, H7 and H10), our analyses provided further detail. Haplogroup dispersal and representation was (necessarily) random, but revealed that the highly prevalent haplogroup $\mathrm{H} 1[20,44,59]$ - not tested in earlier pan-Italian mtDNA population studies $[60,61]$ - is composed of several sublineages (Fig. 2 and Fig. 3). This pilot study lays the scientific foundation for an extended follow-up project to analyze the highresolution phylogeny and phylogeography of the most common West Eurasian CR haplotype in Italy (and beyond). Such data is expected to allow a molecular dissection into sub-clades of more restricted geographic distribution - a possible forensic investigative lead - and younger ages, which will aid to reconstruct migration history $(c f .[20,23])$. Together with insights from other lineages, substructure and stratification revealed could help to establish currently lacking population genetic correction factors for forensic statistics [17].

\section{Conclusion and outlook}

This study makes the forensic (mito-)geneticist's ultimate desire come true: to discern the "identical" by entering the final genetic phase of mtDNA resolution, the analysis of entire mtGenomes. However, MPS appears - for now - out of reach for most forensic casework laboratories, despite its clear advantages in terms of discrimination power, heteroplasmy detection and phylogenetic assignment, which can act as quality control ( $c f$. $[7,49]$ ). Complete mtGenome Sanger-type sequencing is tedious [31,32], even though large-scale automation protocols for use in forensic environment have been presented [6]. Also, legal and ethical questions of (routine) complete mtGenome sequencing need to be resolved [14]. For the time being, an extended version of this study could thus yield specific SNP panels for (forensic) testing of (Italian) samples exhibiting the most common West Eurasian mtDNA CR haplotype. Combined with panels covering other West
Eurasian lineages (e.g., $[29,35,44,62])$, they can serve as a tool ready-to-use for rapid discrimination between divergent lineages in forensic casework or when increased resolution is desirable for investigative purposes but complete $\mathrm{mtGenome} \mathrm{sequencing} \mathrm{is} \mathrm{not}$ feasible. Modular marker sets for hierarchical typing, and "geographic" sets that take the lineages' dispersal into account can be tailored. It should be noted that mtDNA SNP typing can also be a successful alternative in case regular sequencing fails [63].

As a final remark, it remains elusive and beyond the scope of this study to understand why the most common CR motif is so widespread among the radiating haplogroup $\mathrm{H}$ clades regardless of the high mutation rate. Contributing factors may include a founder effect; selective advantages or restrictions favoring an mtDNA population carrying this particular motif, possibly because of functional importance of certain non-coding nucleotide variants; or the high presence of an identical small fraction of the entire molecule could simply be due to the fact that $\mathrm{H}$ is the most common mtDNA haplogroup with the highest number of subclades among the currently overrepresented Western Eurasian entire mtGenomes. Any hypothesis would need to be tested through a comparative analysis of another mtDNA haplogroup on a completely different population background.

\section{Acknowledgments}

The authors wish to thank the donors who gave their blood for research and the two anonymous reviewers for their helpful comments. This work was supported by the intramural funding program of the Medical University Innsbruck for young scientists MUI-START, Project 2013042025, the Theodor Körner Fonds zur Förderung von Wissenschaft und Kunst, the European Union Seventh Framework Programme (FP7/2007-2013) under grant agreement $\mathrm{n}^{\circ} 285487$ and the Austrian Science Fund (FWF) [P22880-B12].

\section{Appendix A. Supplementary data}

Supplementary data associated with this article can be found, in the online version, at doi:10.1016/j.fsigen.2014.09.012. 


\section{References}

[1] J.M. Butler, Fundamentals of Forensic DNA Typing, Elsevier Academic Press, San Diego and London, 2010

[2] C. Eichmann, W. Parson, 'Mitominis': multiplex PCR analysis of reduced size amplicons for compound sequence analysis of the entire mtDNA control region in highly degraded samples, Int. J. Legal Med. 122 (5) (2008) 385-388.

[3] M.D.Coble,TheidentificationoftheRomanovs:canwe(finally)putthecontro-versies torest? Investig.Genet.2(1)(2011)20.

[4] E.Ziętkiewicz,M.Witt,P.Daca,JŻebracka-Gala,M.Goniewicz,B.Jarząb, M.Witt, Currentgeneticmethodologiesintheidentificationofdisastervictimsandin forensic analysis,J.Appl.Genet.53(2012)41-60.

[5] C.M. Bauer, M. Bodner, H. Niederstätter, D. Niederwieser, G. Huber, P. HatzerGrubwieser, K. Holubar, W. Parson, Molecular genetic investigations on Austria's patron saint Leopold III, Forensic Sci. Int. Genet. 7 (2) (2013) 313-315.

[6] R.S. Just, M.K. Scheible, S.A. Fast, K. Sturk-Andreaggi, J.L. Higginbotham, E.A. Lyons, J.M. Bush, M.A. Peck, J.D. Ring, T.M. Diegoli, et al., Development of forensic-quality full $\mathrm{mtGenome} \mathrm{haplotypes:} \mathrm{success} \mathrm{rates} \mathrm{with} \mathrm{low} \mathrm{template} \mathrm{specimens,} \mathrm{Forensic}$ Sci. Int. Genet. 10 (2014) 73-79.

[7] W. Parson, H.J. Bandelt, Extended guidelines for mtDNA typing of population data in forensic science, Forensic Sci. Int. Genet. 1 (1) (2007) 13-19.

[8] H.J. Bandelt, W. Parson, Consistent treatment of length variants in the human mtDNA control region: a reappraisal, Int. J. Legal Med. 122 (1) (2008) 11-21.

[9] A. Salas, H.J. Bandelt, V. Macaulay, M.B. Richards, Phylogeographic investigations: the role of trees in forensic genetics, Forensic Sci. Int. 168 (1) (2007) 1-13.

[10] J.A. Irwin, W. Parson, M.D. Coble, R.S. Just, MtGenome reference population databases and the future of forensic mtDNA analysis, Forensic Sci. Int. Genet. 5 (3) (2011) 222-225

[11] K.A. Sturk, M.D. Coble, S.M. Barritt, T.J. Parsons, R.S. Just, The application of mtDNA SNPs to a forensic case, Forensic Sci. Int. Genet. Suppl. Ser. 1 (1) (2008) 295-297.

[12] R.S. Just, O.M. Loreille, J.E. Molto, D.A. Merriwether, S.R. Woodward, C. Matheson, J. Creed,S.E.McGrath,K.Sturk-Andreaggi,M.D.Coble,etal.,Titanic'sunknown child: thecriticalroleofthemitochondrialDNAcodingregioninare-identifica-tioneffort, ForensicSci.Int.Genet.5(3)(2011)231-235.

[13] S. Lutz, H. Wittig, H.J. Weisser, J. Heizmann, A. Junge, N. Dimo-Simonin, W. Parson, J. Edelmann, K. Anslinger, S. Jung, et al., Is it possible to differentiate mtDNA by means of HVIII in samples that cannot be distinguished by sequencing the HVI and HVII regions? Forensic Sci. Int. 113 (1-3) (2000) 97-101.

[14] M.D. Coble, R.S. Just, J.E. O'Callaghan, I.H. Letmanyi, C.T. Peterson, J.A. Irwin, T.J Parsons, Single nucleotide polymorphisms over the entire mtDNA genome that increase the power of forensic testing in Caucasians, Int. J. Legal Med. 118 (3) (2004) 137-146.

[15] M. van Oven, M. Kayser, Updated comprehensive phylogenetic tree of globa human mitochondrial DNA variation, Hum. Mutat. 30 (2) (2009) E386-E394.

[16] G.Tully,W.Bär,B.Brinkmann,A.Carracedo,P.Gill,N.Morling,W.Parson,P. Schneider, ConsiderationsbytheEuropeanDNAprofiling(EDNAP)grouponthe working practices,nomenclatureandinterpretationofmitochondrialDNApro-files, Forensic Sci.Int.124(1)(2001)83-91.

[17] ScientificWorkingGrouponDNAAnalysisMethods(SWGDAM),InterpretationGuidelinesforMitochondrialDNAAnalysisbyForensicDNATestingLaboratories,2013.

[18] B.A. Malyarchuk, T. Grzybowski, M.V. Derenko, J. Czarny, M. Woźniak, D. Miścicka-Sliwka, Mitochondrial DNA variability in Poles and Russians, Ann. Hum. Genet. 66 (2002) 261-283.

[19] S. Cardoso, M.A. Alfonso-Sánchez, L. Valverde, D. Sánchez, M.T. Zarrabeitia, A. Odriozola, B. Martínez-Jarreta,M.M.dePancorbo,GeneticuniquenessoftheWaoranitribefrom the EcuadorianAmazon,Heredity(Edinb.)108(6)(2012)609-615.

[20] A.Achilli,C.Rengo,C.Magri,V.Battaglia,A.Olivieri,R.Scozzari,F.Cruciani,M. Zeviani,E. Briem,V.Carelli,etal.,ThemoleculardissectionofmtDNAhaplogroup Hconfirmsthat theFranco-Cantabrianglacialrefugewasamajorsourceforthe Europeangenepool, Am.J.Hum.Genet.75(5)(2004)910-918.

[21] A. Brandstätter, B. Zimmermann, J. Wagner, T. Göbel, A.W. Röck, A. Salas, A. Carracedo W. Parson, Timing and deciphering mitochondrial DNA macro-haplogroup R0 variability in Central Europe and Middle East, BMC Evol. Biol. 8 (2008) 191.

[22] W. Parson, A. Dür, EMPOP - a forensic mtDNA database, Forensic Sci. Int. Genet. 1 (2) (2007) 88-92, Available at: http://empop.org.

[23] E.L. Loogväli, U. Roostalu, B.A. Malyarchuk, M.V. Derenko, T. Kivisild, E. Metspalu, K Tambets, M. Reidla, H.V. Tolk, J. Parik, et al., Disuniting uniformity: a pied cladistic canvas of mtDNA haplogroup H in Eurasia, Mol. Biol. Evol. 21 (11) (2004) 2012-2021.

[24] A. Brandstätter, A. Salas, H. Niederstätter, C. Gassner, A. Carracedo, W. Parson, Dissection of mitochondrial superhaplogroup $\mathrm{H}$ using coding region SNPs, Electrophoresis 27 (13) (2006) 2541-2550.

[25] U. Roostalu, I. Kutuev, E.L. Loogväli, E. Metspalu, K. Tambets, M. Reidla, E.K. Khusnutdinova, E. Usanga, T. Kivisild, R. Villems, Origin and expansion of haplogroup $\mathrm{H}$, the dominant human mitochondrial DNA lineage in West Eurasia: the Near Eastern and Caucasian perspective, Mol. Biol. Evol. 24 (2) (2007) 436-448.

[26] V. Álvarez-Iglesias, A. Mosquera-Miguel, M. Cerezo, B. Quintáns, M.T. Zarrabeitia I. Cuscó,M.V.Lareu,O.García,L.Pérez-Jurado,A.Carracedo,etal.,Newpopulation and phylogeneticfeaturesoftheinternalvariationwithinmtDNAmacroha-plogroupRo, PLoSONE4(4)(2009)e5112.

[27] D.M. Behar, M. van Oven, S. Rosset, M. Metspalu, E.L. Loogväli, N.M. Silva, T. Kivisild, A. Torroni, R. Villems, A "Copernican" reassessment of the human mitochondrial DNA tree from its root, Am. J. Hum. Genet. 90 (4) (2012) 675-684.

[28] R.M. Andrews, I. Kubacka, P.F. Chinnery, R.N. Lightowlers, D.M. Turnbull, N Howell, Reanalysis and revision of the Cambridge reference sequence for human mitochondrial DNA, Nat. Genet. 23 (2) (1999) 147.
[29] S. Köhnemann, C. Hohoff, H. Pfeiffer, An economical mtDNA SNP assay detecting different mitochondrial haplogroups in identical HVR 1 samples of Caucasian ancestry, Mitochondrion 9 (5) (2009) 370-375.

[30] T.J. Parsons, M.D. Coble, Increasing the forensic discrimination of mitochondrial DNA testing through analysis of the entire mitochondrial DNA genome, Croat. Med. J. 42 (3) (2001) 304-309.

[31] L. Fendt, B. Zimmermann, M. Daniaux, W. Parson, Sequencing strategy for the whole mitochondrial genome resulting in high quality sequences, BMC Genomics 10 (2009) 139.

[32] E.A. Lyons, M.K. Scheible, K. Sturk-Andreaggi, J.A. Irwin, R.S. Just, A high-throughput Sanger strategy for human mitochondrial genome sequencing, BMC Genomics 14 (2013) 881

[33] H. Andréasson, M. Nilsson, H. Styrman, U. Pettersson, M. Allen, Forensic mitochondrial coding region analysis for increased discrimination using pyrosequencing technology, Forensic Sci. Int. Genet. 1 (1) (2007) 35-43.

[34] B. Zimmermann, M. Bodner, S. Amory, L. Fendt, A. Röck, D. Horst, B. Horst, T Sanguansermsri, W. Parson, A. Brandstätter, Forensic and phylogeographic characterization of mtDNA lineages from northern Thailand (Chiang Mai), Int. J. Legal Med. 123 (6) (2009) 495-501.

[35] M. Mikkelsen, E. Rockenbauer, E. Sørensen, M. Rasmussen, C. Børsting, N. Morling A mitochondrial DNA SNP multiplex assigning Caucasians into 36 haplo- and subhaplogroups, Forensic Sci. Int. Genet. Suppl. Ser. 1 (1) (2008) 287-289.

[36] A. Coutinho, G. Valverde, L. Fehren-Schmitz, A. Cooper, M.I. Barreto Romero, I.F. Espinoza, B. Llamas, W. Haak, AmericaPlex26: a SNaPshot multiplex system for genotyping the main human mitochondrial founder lineages of the Americas, PLOS ONE 9 (3) (2014) e93292.

[37] K.Krjutškov,T.Viltrop,P.Palta,E.Metspalu,E.Tamm,S.Suvi,K.Sak,A.Merilo,H. Sork,R. Teek,etal.,Evaluationofthe124-plexSNPtypingmicroarrayforforensic testing, ForensicSci.Int.Genet.4(1)(2009)43-48.

[38] M.vanOven,M.Vermeulen,M.Kayser,Multiplexgenotypingsystemforefficient inferenceofmatrilinealgeneticancestrywithcontinentalresolution,Investig. Genet. 23(2)(2011)6.

[39] L. Chaitanya, M. van Oven, N. Weiler, J. Harteveld, L. Wirken, T. Sijen, P. de Knijff, M. Kayser, Developmental validation of mitochondrial DNA genotyping assays for adept matrilineal inference of biogeographic ancestry at a continental level, Forensic Sci. Int. Genet. 11 (2014) 39-51.

[40] L. Pereira, M. Richards, A. Goios, A. Alonso, C. Albarrán, O. Garcia, D.M. Behar, M. Gölge, J. Hatina, L. Al-Gazali, et al., Evaluating the forensic informativeness of mtDNA haplogroup H sub-typing on a Eurasian scale, Forensic Sci. Int. 159 (1) (2006) 43-50

[41] P.Grignani,G.Peloso,A.Achilli,C.Turchi,A.Tagliabracci,M.Alù,G.Beduschi,U. Ricci,L. Giunti,C.Robino,etal.,SubtypingmtDNAhaplogroupHbySNaPshot minisequencing anditsapplicationinforensicindividualidentification,Int.J. LegalMed.120(3)(2006) $151-156$.

[42] M. Nilsson, H. Andréasson-Jansson, M. Ingman, M. Allen, Evaluation of mitochondrial DNA coding region assays for increased discrimination in forensic analysis, Forensic Sci. Int. Genet. 2 (1) (2008) 1-8.

[43] A. Mosquera-Miguel, V. Álvarez-Iglesias, M. Cerezo, M.V. Lareu, A. Carracedo, A. Salas, Testing the performance of mtSNP minisequencing in forensic samples, Forensic Sci. Int. Genet. 3 (4) (2009) 261-264.

[44] A. Boattini, B. Martinez-Cruz, S. Sarno, C. Harmant, A. Useli, P. Sanz, D. YangYao, J. Manry, G. Ciani, D. Luiselli, et al., Uniparental markers in Italy reveal a sex-biased genetic structure and different historical strata, PLOS ONE 8 (5) (2013) e65441.

[45] H.J. Bandelt, A. Salas, Current next generation sequencing technology may not meet forensic standards, Forensic Sci. Int. Genet. 6 (1) (2012) 143-145.

[46] W. Parson, C. Strobl, G. Huber, B. Zimmermann, S.M. Gomes, L. Souto, L. Fendt, R. Delport, R. Langit, S. Wootton, et al., Evaluation of next generation mtGenome sequencing using the Ion Torrent Personal Genome Machine (PGM), Forensic Sci. Int. Genet. 7 (5) (2013) 543-549.

[47] B.Zimmermann,A.Röck,G.Huber,T.Krämer,P.M.Schneider,W.Parson, Application ofawestEurasian-specificfilterforquasi-mediannetworkanalysis: sharpeningthe bladeformtDNAerrordetection,ForensicSci.Int.Genet.5(2)(2011)133-137.

[48] R.S. Just, S.A. Fast, M.K. Scheible, K. Sturk-Andreaggi, A.W. Röck, J.M. Bush, J.L. Higginbotham, M.A. Peck, J.D. Ring, G.E. Huber, et al., Full mtGenome referencedata: development and characterization of 588 forensic-quality haplotypesrepresenting three U.S. populations, Forensic Sci. Int. Genet. (2014), http:// dx.doi.org/10.1016/j.fsigen.2014.09.021.

[49] J.L. King, B.L. LaRue, N.M. Novroski, M. Stoljarova, S.B. Seo, X. Zeng, D.H. Warshauer, C.P. Davis, W. Parson, A. Sajantila, et al., High-quality and high-throughput massively parallel sequencing of the human mitochondrial genome using the Illumina MiSeq, Forensic Sci. Int. Genet. 12C (2014) 128-135.

[50] S.A. Miller, D.D. Dykes, H.F. Polesky, A simple salting out procedure for extracting DNA from human nucleated cells, Nucleic Acids Res. 16 (1988) 1215

[51] H. Niederstätter, S. Köchl, P. Grubwieser, M. Pavlic, M. Steinlechner, W. Parson, A modular real-time PCR concept for determining the quantity and quality of human nuclear and mitochondrial DNA, Forensic Sci. Int. Genet. 1 (1) (2007) 29-34.

[52] A.W. Röck, A. Dür, M. van Oven, W. Parson, Concept for estimating mitochondrial DNA haplogroups using a maximum likelihood approach (EMMA), Forensic Sci. Int. Genet. 7 (6) (2013) 601-609.

[53] M.Stoneking,D.Hedgecock,R.G.Higuchi,L.Vigilant,H.A.Erlich,Population variation ofhumanmtDNAcontrolregionsequencesdetectedbyenzymatic amplificationand sequence-specificoligonucleotideprobes,Am.J.Hum.Genet. 48(2)(1991)370-382. 
[54] C.Fraumene,E.M.Belle,L.Castrì,S.Sanna,G.Mancosu,M.Cosso,F.Marras,G. Barbujani,M.Pirastu,A.Angius,Highresolutionanalysisandphylogenetic network constructionusingcompletemtDNAsequencesinSardiniangenetic isolates, Mol. Biol.Evol.23(11)(2006)2101-2111.

[55] C. Herrnstadt, J.L. Elson, E. Fahy, G. Preston, D.M. Turnbull, C. Anderson, S.S. Ghosh, J.M. Olefsky, M.F. Beal, R.E. Davis, et al., Reduced-median-network analysis of complete mitochondrial DNA coding-region sequences for the major African, Asian, and European haplogroups, Am. J. Hum. Genet. 70 (5) (2002) 1152-1171.

[56] N.Raule,F.Sevini,S.Li,A.Barbieri,F.Tallaro,L.Lomartire,D.Vianello,A. Montesanto,J.S. Moilanen,V.Bezrukov,etal.,Theco-occurrenceofmtDNA mutationsondifferent oxidativephosphorylationsubunits,notdetectedby haplogroupanalysis,affects humanlongevityandispopulationspecific,Aging Cell13(3)(2014)401-407.

[57] G. Gasparre, A.M. Porcelli, E. Bonora, L.F. Pennisi, M. Toller, L. Iommarini, A. Ghelli, M. Moretti, C.M. Betts, G.N. Martinelli, et al., Disruptive mitochondrial DNA mutations in complex I subunits are markers of oncocytic phenotype in thyroid tumors, Proc. Natl. Acad. Sci. U. S. A. 104 (21) (2007) 9001-9006.
[58] M. Bodner, J.A. Irwin, M.D. Coble, W. Parson, Inspecting close maternal relatedness: towards better mtDNA population samples in forensic databases, Forensic Sci. Int. Genet. 5 (2) (2011) 138-141.

[59] F. Brisighelli, V. Álvarez-Iglesias, M. Fondevila, A. Blanco-Verea, A. Carracedo, V.L. Pascali, C. Capelli, A. Salas, Uniparental markers of contemporary Italian population reveals details on its pre-Roman heritage, PLOS ONE 7 (12) (2012) e50794.

[60] A. Tagliabracci, C. Turchi, L. Buscemi, C. Sassaroli, Polymorphism of the mitochon-drial DNA control region in Italians, Int. J. Legal Med. 114 (4-5) (2001) 224-228

[61] C.Turchi,L.Buscemi,C.Previderè,P.Grignani,A.Brandstätter,A.Achilli,W. Parson,A. Tagliabracci,Ge.F.I.Group,ItalianmitochondrialDNAdatabase:results ofa collaborativeexerciseandproficiencytesting,Int.J.LegalMed.122(2008) 199-204.

[62] A.Brandstätter,T.J.Parsons,W.Parson,RapidscreeningofmtDNAcodingregion SNPs fortheidentificationofwestEuropeanCaucasianhaplogroups,Int.J.Legal Med.117 (5)(2003)291-298

[63] S. Köhnemann, H. Pfeiffer, Application of mtDNA SNP analysis in forensic casework, Forensic Sci. Int. Genet. 5 (3) (2011) 216-221 